\section{Evaluación problemática ambiental asociada a comunidad aledaña a institución de educación superior en Cartagena. Enfoque de participación comunitaria}

\author{
Environmental evaluation associated to community close \\ Higher Education Institution in Cartagena. \\ Community Involvement Approach
}

Recibido: 15-feb-17 - Revisado: 30-abr-17

Aceptado: 30-may-17 - Publicado: 30-jul-17

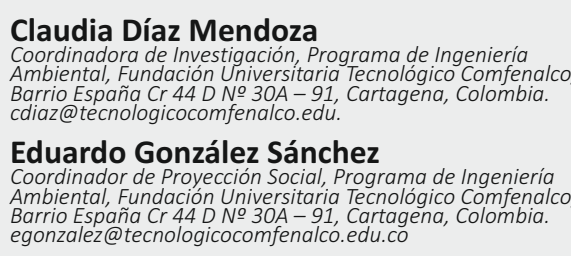

Dendoza

Co - 91. Cartagena, Colombia.

Eduardo González Sánchez Barrio España Cr 44 D № 30A - 91, Cartagena, Colombia. egonzalez@tecnologicocomfenalco.edu.co

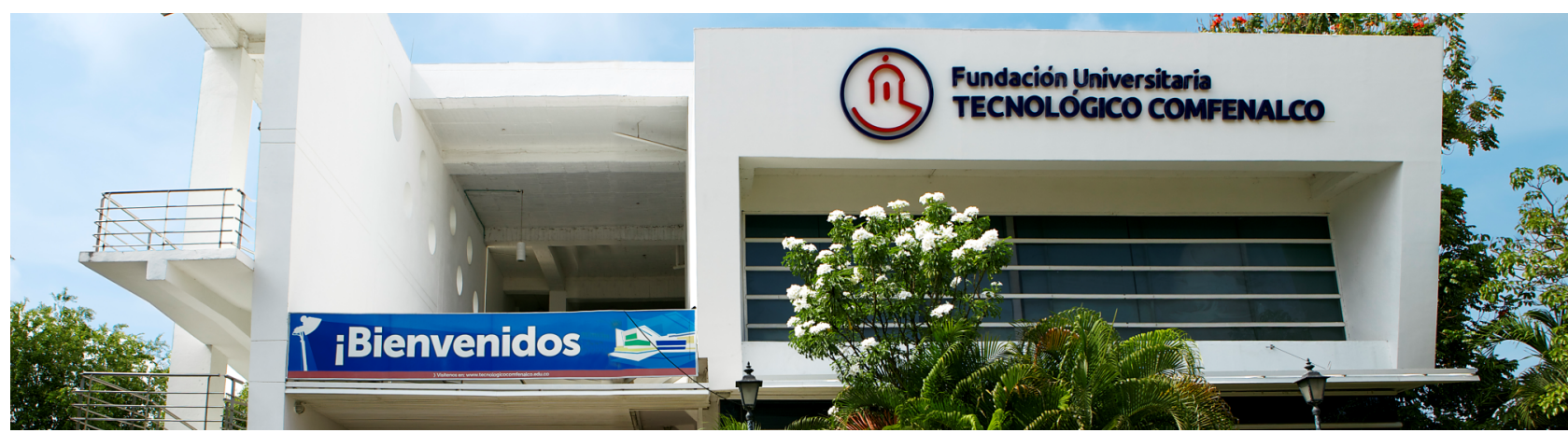

Resumen: Los proyectos de educación ambiental deben ser incluidos de forma transversal en las instituciones educativas, desde la etapa escolar hasta las instituciones de educación superior; de igual forma, la responsabilidad con el ambiente debe proyectarse en la academia y en especial en las instituciones de educación superior hacia las comunidades locales y/o regionales. Es pertinente que en materia ambiental, se evalúen problemas contextualizados y, precisamente, son las propias comunidades quienes poseen información primaria de los aspectos ambientales con los que conviven. Por ello, el objetivo del proyecto fue identificar la problemática ambiental asociada a la comunidad aledaña a la Fundación Universitaria Tecnológico Comfenalco, Cartagena, a partir de una metodología de participación comunitaria, en la cual la comunidad aportó su conocimiento histórico de los aspectos ambientales en la zona. Adicionalmente, se comparó la información obtenida con un estudio anteriormente realizado en el marco del proyecto de «Fortalecimiento de la Gestión Ambiental de Cartagena». Como principales resultados, se evidencian los aspectos ambientales identificados como significativos de carácter negativo: ruido, manejo inadecuado de residuos sólidos y falta de arborización.

Palabras clave: Aspectos ambientales, comunidad, participativo, proyectos.

Abstract: Environmental Education projects should be included transversally in educational institutions from the school to University, just as responsibility for the environment must be projected from academia and especially from higher education institutions to Local and / or regional communities; Since it is pertinent that in environmental matters contextualized problems are evaluated and it is the communities themselves that possess primary information of the environmental aspects with which they coexist. The objective of the project was to identify the environmental problems associated with the community surrounding the Fundación Universitaria Tecnológico Comfenalco Cartagena, based on a methodology of community participation, in which the community contributed its historical knowledge of the environmental aspects in the area, additional Compared the information obtained with an earlier study of the Cartagena Environmental Management project. As main results are evidenced, the environmental aspects identified as significant of negative character: noise, inadequate solid waste management and lack of afforestation.

Key words: Environmental aspects, community, intervene, projects. 


\section{INTRODUCCIÓN}

La educación ambiental latinoamericana se construyó a partir de las experiencias de la educación popular, de la educación comunitaria y participativa y de la educación ecológica o conservacionista, en su paso hacia una educación ambiental comprometida con el cambio social y con la transformación de los modelos económicos de desarrollo (Tréllez, 2006).

La adquisición de una conciencia para la conservación, protección y mejoramiento del ambiente, de la calidad de la vida, del uso racional de los recursos naturales, así como la enseñanza de la protección del ambiente y la preservación de los recursos naturales, de conformidad con lo establecido en el artículo 67 de la Constitución Política colombiana, debe ser tema de interés para las instituciones de educación superior. Por lo tanto, es importante identificar las principales problemáticas ambientales asociadas a las comunidades para establecer mecanismos adecuados que logren garantizar la sostenibilidad ambiental de las mismas a partir de un diagnóstico participativo. La Investigación

Acción Participativa constituye una opción metodológica de mucha riqueza, ya que, por una parte, permite la expansión del conocimiento y por la otra, genera respuestas concretas a problemáticas que se plantean los investigadores y coinvestigadores cuando deciden abordar un interrogante o situación problemática y desean aportar alguna alternativa de cambio o transformación (Colmenares, 2012). Las comunidades que participan en la identificación de sus problemáticas, en especial las ambientales, y que proponen los proyectos de intervención crean mayor empoderamiento con las soluciones propuestas. Asimismo, los actores sociales se convierten en investigadores activos, participando en la identificación de las necesidades o los potenciales problemas por investigar, en la recolección de información, en la toma de decisiones, en los procesos de reflexión y acción (Colmenares, 2012).
El monitoreo y la evaluación participativa en los procesos de investigación se consolidan como el cambio del enfoque metodológico, en el cual la comunidad, los donantes y decidores de políticas en conjunto eligen cómo medir el progreso de los proyectos y qué acciones implementar para mantener y mejorar los resultados (Mistelli et al., 2009). Por lo anterior, se establece como objetivo de la investigación: la identificación de las problemáticas ambientales presentadas por la comunidad y la propuesta de proyectos que mitiguen los aspectos ambientales significativos de carácter negativos identificados.

\section{METODOLOGÍA}

El proyecto corresponde a una investigación evaluativa. Esta inicia con el estadio descriptivo en el que se pretende valorar las condiciones ambientales existentes en la comunidad, en cuanto a condiciones existentes y conocimientos en temas ambientales importantes para la conservación de los recursos.

La metodología del proyecto se estructuró por fases. En la primera, se determinó la población objeto de estudio, el área de influencia del proyecto mediante uso de sistema de información geográfica y mediante muestreo aleatorio simple, se trabajó de forma participativa. En la segunda fase se realizó el diseño del instrumento a aplicar, de acuerdo a los aspectos ambientales y sociales a identificar; se llevó a cabo la recolección de la información a través de la participación activa de la comunidad en la identificación de los problemas asociados a su entorno de acuerdo al instrumento diseñado. A continuación, se desarrolló el análisis estadístico de la información, evaluando la relación con la información previa obtenida del estudio de percepción ambiental por comunidades realizado en el 2015. Como fase final, el proyecto contempla la socialización de resultados con la comunidad y el diseño de estrategias derivadas de los aspectos identificados. 


\section{Trisuos}

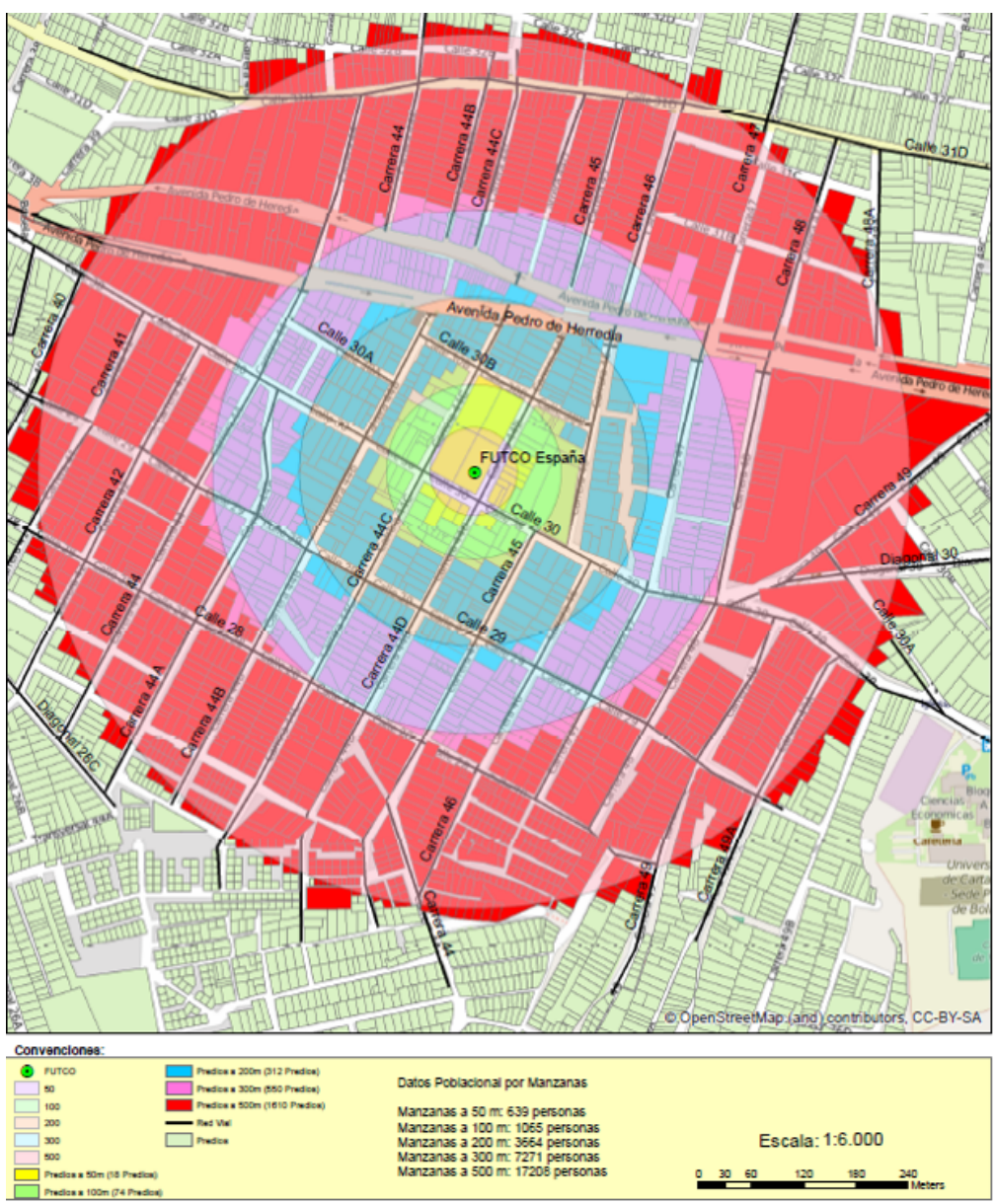

Fig. 1. Localización de predios zona aledaña a Sede A.

Fuente: Payares, 2015.

\section{RESULTADOS Y DISCUSIÓN}

El proyecto se desarrolla teniendo en cuenta la localización de la Sede A, Barrio España, carrera 44D \# 30 A-91, de la Fundación Universitaria Tecnológico Comfenalco, en Cartagena. Se llevó a cabo una modelación en sistema de información geográfica con datos poblacionales de $50 \mathrm{~m}, 100 \mathrm{~m}, 200 \mathrm{~m}, 300 \mathrm{~m}$ y $500 \mathrm{~m}$. Así, se obtuvo una distribución de predios como la mostrada en la Fig. 1, con un aproximado de 17208 personas y un aproximado de 1610 predios.

Teniendo como suposiciones estadísticas que cada encuesta será independiente, que no existe información previa de este tipo de estudio, que los datos de estudio se distribuirán de manera normal, que la estadística paramétrica posee un margen de error del $5 \%$ y un nivel de confianza de $95 \%$; se diseñó un muestreo aleatorio simple, verificando el cálculo de error muestral para poblaciones finitas e infinitas. De este modo, se determinó un tamaño de muestra de 310 predios a encuestar.

Como dato relevante, se reporta que el porcentaje de aceptación del instrumento estadístico es del $95 \%$. El uso de los predios de las personas participantes en el proyecto fue de un $74 \%$ en cuestión de viviendas y un 
$26 \%$, en cuanto a locales comerciales. El sector incluido en el estudio, de acuerdo a la división político administrativa de Cartagena de Indias (fuente: Secretaría de Planeación Distrital), está ubicado en la localidad Histórica y del Caribe Norte, Unidad Comunera de Gobierno 9. La distribución de los porcentajes de encuestas realizadas por Barrios se indica en la Fig.2.

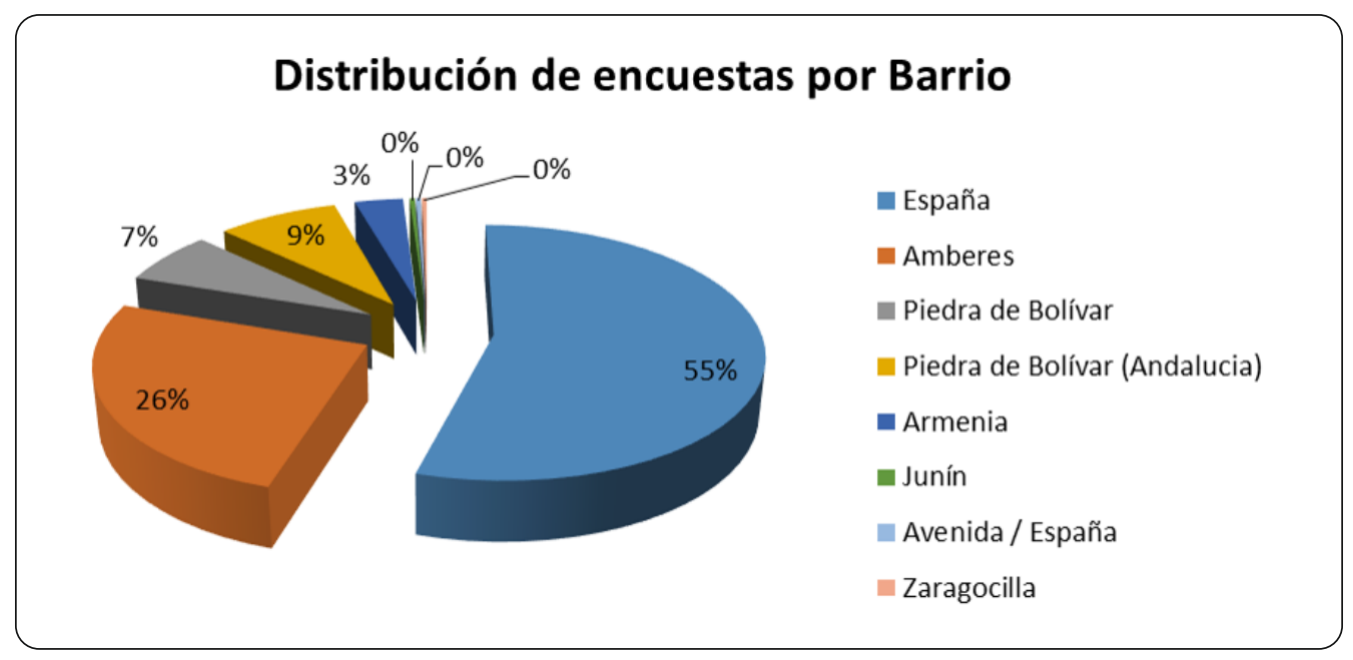

Fig. 2. Distribución por barrios participantes en el proyecto.

Otro aspecto social evaluado es el correspondiente al nivel de formación y el tiempo de residencia o de realizar actividades en la zona. La información derivada de estos dos aspectos se muestra en las figuras 3 y 4 , donde se observa el máximo nivel de formación de las personas participantes en el proceso. Como resultados destacados, el nivel predominante fue bachiller, seguido por técnico y primaria. Es importante resaltar el alto nivel de personas con grado de escolaridad de bachiller en una zona donde hay más de tres instituciones universitarias en el área de influencia.

De acuerdo a los informes del Ministerio de Educación Nacional (MEN), en Colombia cada vez más jóvenes tienen la posibilidad de acceder a la educación superior; al revisar la cobertura bruta de hace 10 años, se halla que esta se encontraba en $25,8 \%$ y hoy llega al $46,1 \%$ de la población entre 17 y 21 años.

Esta tendencia ha significado un avance fundamental en materia de equidad, si se tiene en cuenta que el 60 $\%$ de los estudiantes nuevos que están ingresando a algún programa de educación superior provienen de familias con ingresos inferiores a dos salarios mínimos (Dúran Muriel, 2015).

Por los resultados obtenidos, sería importante ampliar el estudio y revisar los rangos de edades de las personas participantes. 


\section{Firmos}

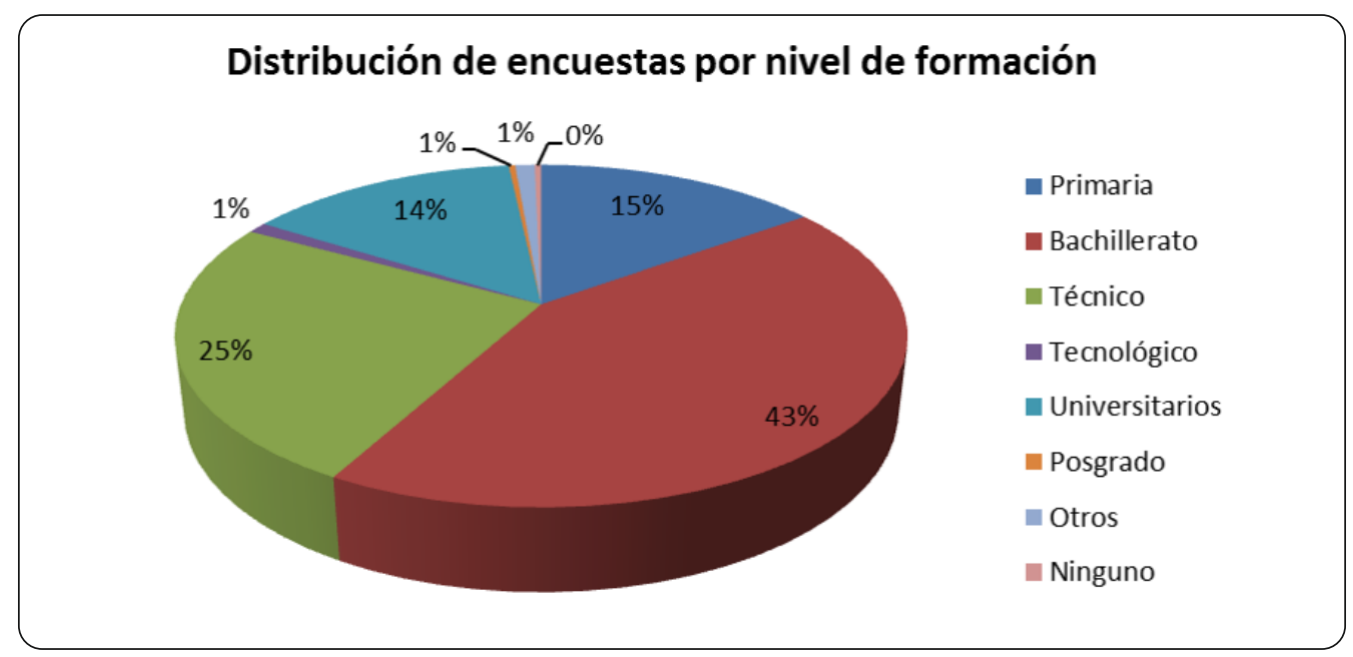

Fig. 3. Distribución por máximo nivel de formación.

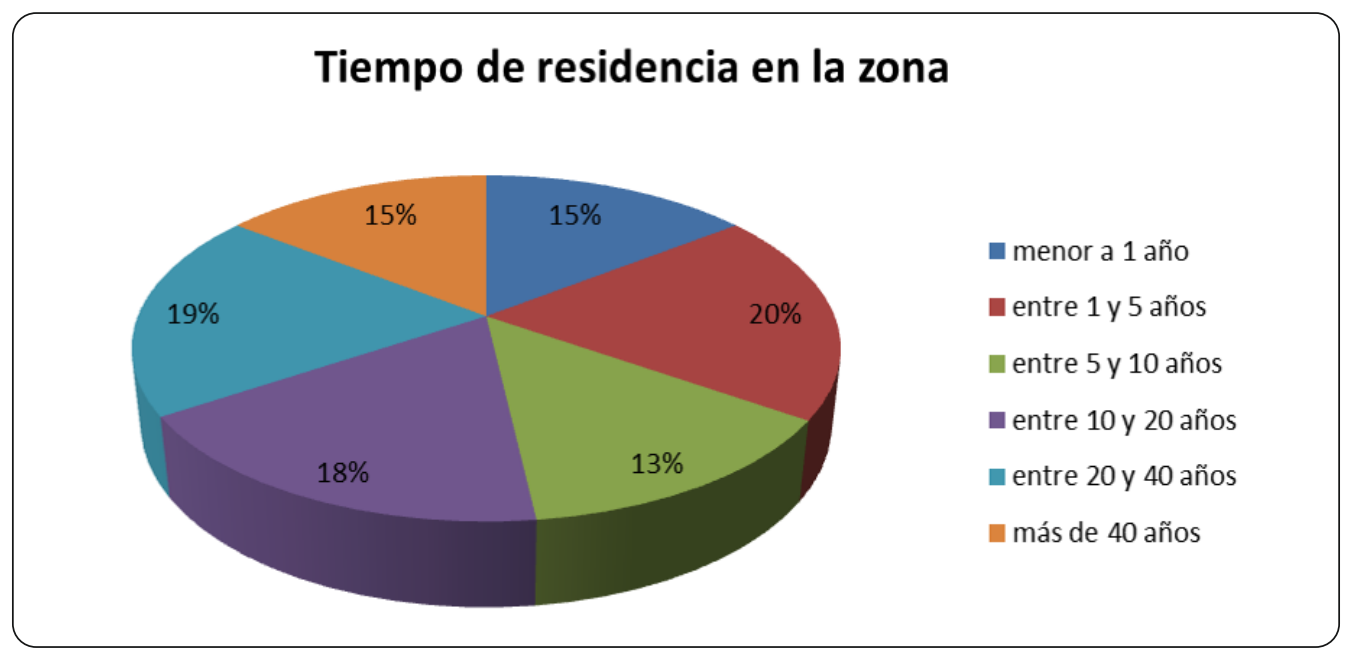

Fig. 4. Distribución de participación por tiempos de residencia en la zona.

La Fig. 4 muestra que la mayoría de las personas participantes de la muestra (65\%) tienen tiempos de residencia en la zona mayores a cinco años, lo cual es un factor positivo para el proyecto porque son individuos que, gracias a los largos tiempos de estadía en la zona, pueden percibir con mayor asertividad los problemas ambientales de sus comunidades y los aportes en la identificación de línea base se reportan con mayor precisión.
Ahora bien, los aspectos que identifica cada individuo como negativos son percibidos por los mismos como aquellos factores que afectan su calidad de vida. Este concepto es ampliamente definido por Cummins (2000); luego de un extenso análisis sobre lo subjetivo y lo objetivo en las mediciones en $\mathrm{CV}$, concluye que ambos tipos de medidas han demostrado ser indicadores útiles y aun cuando son independientes, el grado de dependencia aumenta cuando las condiciones objetivas de vida son más pobres. 
Sobre lo mismo, Wrosch \& Scheier (2003) y Huppert \& Whittington (2003) plantean que se ha investigado la CV relacionándola con indicadores objetivos (condiciones de vida, estado de salud) e indicadores subjetivos (satisfacción con la vida, bienestar). Ambos explican parte de la varianza de la CV reportada por las personas y afirman que las personas pueden valorar más una cosa que otra, dependiendo de la significación que le otorguen y los estándares de comparación.

Los aspectos ambientales identificados por la comunidad como impactos significativos al ambiente con carácter negativo se muestran en distribución numérica en la Fig. 5, donde se aprecia que los mayores aspectos identificados corresponden a alta sensación térmica, poca arborización y ruido en la zona.

En cuanto a la sensación o confort térmico, Moreno \& Fernández (2003) indican que de las múltiples variables físico-ambientales que inciden sobre el hombre, las relacionadas con el clima ocupan un lugar dominante, debido, por un lado, al carácter cambiante de las situaciones atmosféricas y por otro, a las características fisiológicas del organismo humano: los frecuentes cambios en la presión atmosférica, la humedad y la temperatura someten al organismo a un continuo proceso de adaptación que necesariamente condiciona el grado de confort y bienestar del ambiente en el que el hombre vive y desarrolla sus actividades. La vegetación de mayor incidencia en este proceso de mejoramiento de las condiciones ambientales es la arbórea, pues amortigua los efectos de la radiación solar, modera la temperatura de su entorno inmediato, incrementa o disminuye los niveles de humedad y modifica el comportamiento de los vientos (Jiménez, 2008).

Según Martínez (2005), uno de los principales problemas de las ciudades modernas es el crecimiento del tráfico urbano; su incremento eleva las externalidades ambientales como el ruido, la polución, los desechos tóxicos, entre otras. Las externalidades aumentan en la medida que crecen las ciudades.

El ruido por tráfico urbano, por su parte, está relacionado con el aumento del número de automóviles, camiones, motocicletas, tracto mulas y demás vehículos que circulan por las ciudades. Lo anterior causa afectaciones económicas importantes que tienen dos receptores: los habitantes de la ciudad que reciben el impacto de los niveles de ruido, lo que les genera problemas de salud, y la infraestructura física de la ciudad, en especial el valor de mercado de las propiedades residenciales.

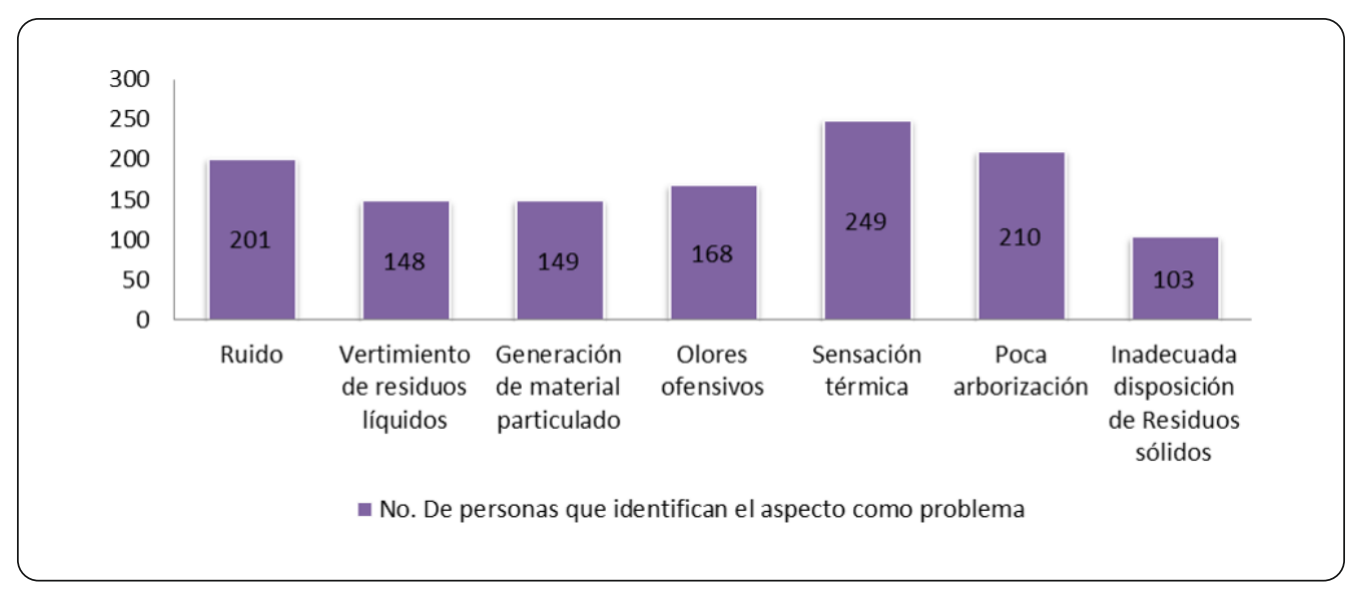

Fig. 5. Distribución numérica de reportes de aspectos ambientales. 
Otro de los aspectos incluidos en el estudio fue la importancia que le otorgaba la comunidad a la realización de talleres de formación ambiental. El 100 $\%$ de las personas participantes respondieron que era muy importante la educación y formación ambiental. A la pregunta orientada a la disposición de cada una de las personas en participar en los talleres ambientales, el $88 \%$ de los encuestados respondió afirmativamente; las personas que respondieron que no participarían atribuían su negativa a la falta de tiempo principalmente y a la edad avanzada de algunas de ellas.

Dentro del ejercicio de participación, a la comunidad, se le solicitó proponer proyectos para ser implementados en su barrio y con los cuales ellos consideraban que se mejoraría la calidad de vida de los habitantes del sector. Los proyectos propuestos se muestran en la Fig. 6, donde se observa que el mayor número de propuestas están encaminadas a la siembra de árboles, para mejorar las condiciones de microclima de la zona; también se le da gran importancia a la limpieza de caños, canales y alcantarillas, ya que se considera que es un factor que genera vectores y olores ofensivos. Otro de los aspectos que destacados son las propuestas de manejo y disposición adecuada de residuos sólidos y proyectos de educación ambiental a la comunidad.

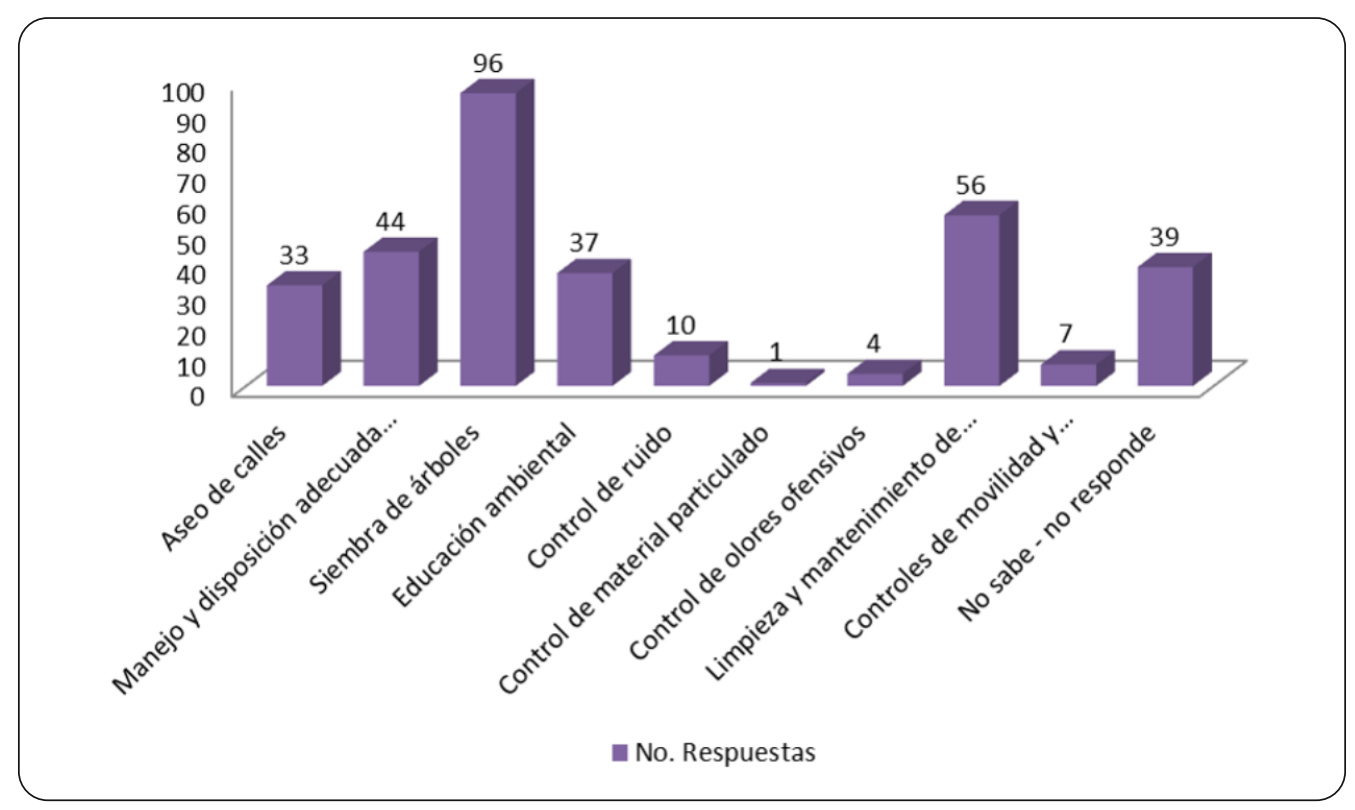

Fig. 6. Propuestas de proyectos ambientales a implementar en la zona de estudio.

Seguidamente, se realizó una comparación con el estudio previo efectuado en el periodo comprendido entre julio y noviembre del 2015; en este último se evaluaban los reportes de una investigación efectuada con reportes hechos por la comunidad de distintos barrios a través de la herramienta informática INVIVO sobre los aspectos que generaban mayor incidencia en trámites ante la autoridad ambiental. En dicha comparación, se encontraron los siguientes datos significativos:

La organización territorial del Distrito de Cartagena contempla tres Localidades: Histórica y del Caribe Norte, De la Virgen y Turística y la localidad Industrial de la Bahía; a su vez, se han agrupado los barrios en 15 unidades comuneras de gobierno. El estudio realizado 
contempló el análisis de participación en el reporte de incidencias ambientales por localidad. En la Fig. 7 se muestra la distribución porcentual de los reportes realizados.

De esta figura, se deduce que la localidad que presenta la mayor incidencia en reportes ambientales es la
Histórica y Caribe Norte, con el 56 \%. Al comparar el reporte mostrado en la Fig. 7 con el estudio desarrollado, se observa que coinciden las zonas de mayores reportes de incidentes ambientales con el reporte de la percepción de la comunidad en estudio, la cual identifica tener aspectos ambientales que inciden en su calidad de vida.

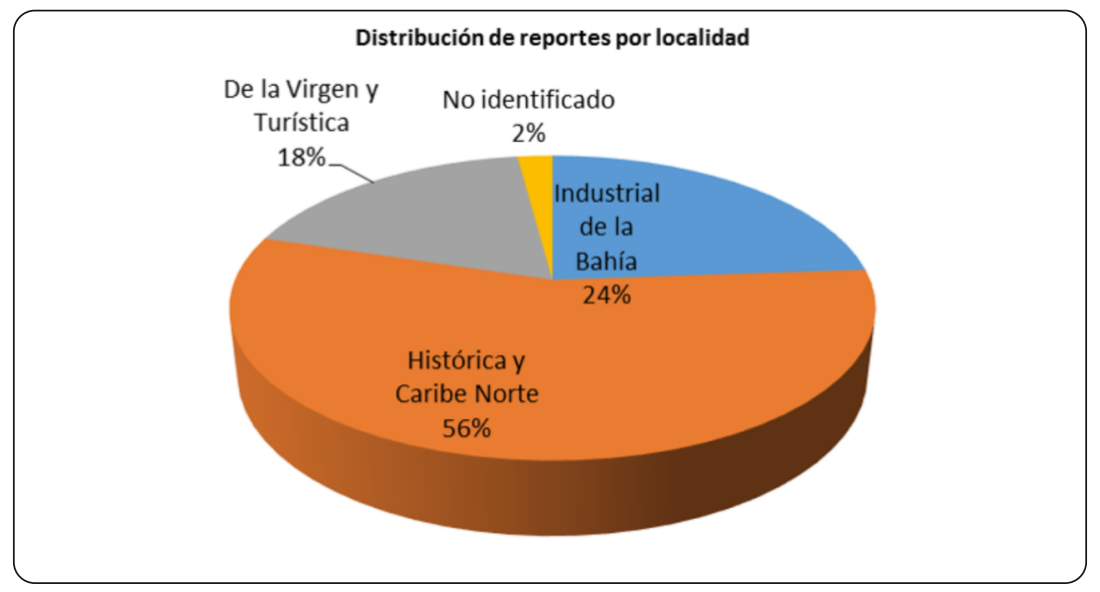

Fig. 7. Distribución de reportes ambientales por Localidad Distrito de Cartagena.

La Fig. 8 muestra el detalle de los reportes con base en el elemento ambiental identificado de acuerdo al estudio del 2015. Esta indica que el mayor porcentaje de los reportes de incidencias ambientales es atribuido a la presencia de basureros a cielo abierto con un $38 \%$, seguido de ruido, con un $20 \%$. Si comparamos con el estudio puntual desarrollado en 2016, notamos que estos dos aspectos ambientales también fueron identificados, en otras palabras, que se corroboran los resultados obtenidos en dos años consecutivos.

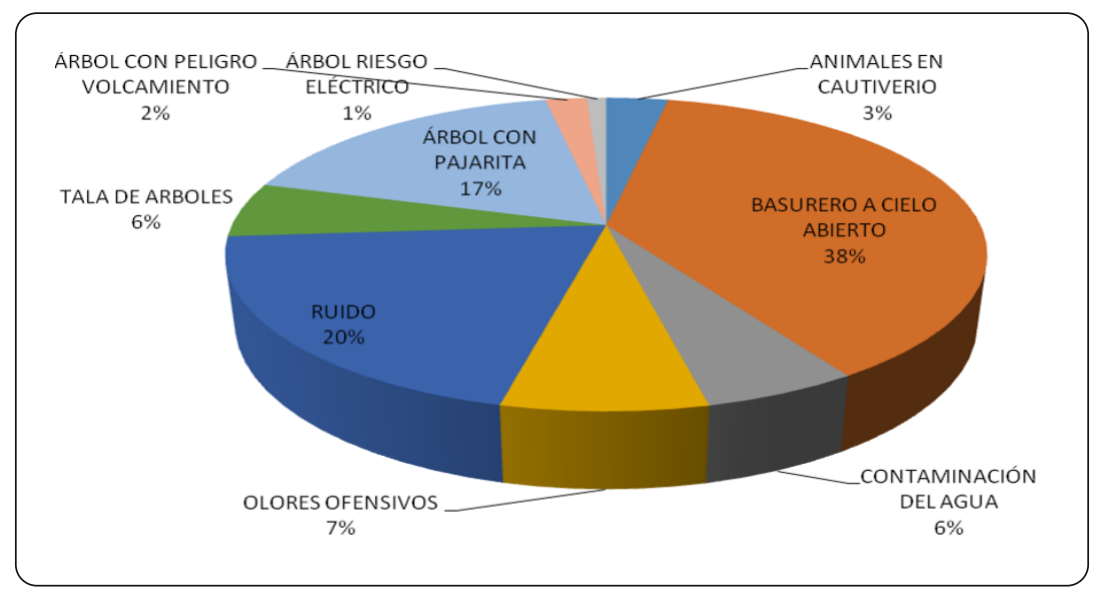

Fig. 7. Distribución porcentual de reportes ambientales 2015. 
Es importante anotar que el proyecto se fundamentó en análisis de percepción; según estudios realizados en mediciones de personas frente a temas ambientales, la mayoría de los grupos estudiados poseen una actitud y valoración positiva hacia el ambiente (Amérigo, Aragonés, De Frutos, Sevillano \& Cortés, 2007; González \& Amérigo, 1999; Hernández, Suarez, Martínez-Tovisco \& Hess, 1997; Milfont, 2010; Pato, Ros \& Tamayo, 2005; Schultz, 2002; TacáksSánta, 2007; Vozmediano \& Guillén, 2005). Sin embargo, tales resultados pueden ser causados por los sesgos que desencadena la deseabilidad social, la cual se entiende como la tendencia que tienen los participantes a dar respuestas que están de acuerdo a lo que la sociedad considera como idóneo (Greenwald, 1990; Sintov \& Prescott, 2011).

Considerando lo anterior, es claro que la percepción ambiental de las personas en torno al ejercicio realizado revela que existe una problemática asociada a falta de arborización, alta sensación térmica y inadecuada disposición de residuos.

\section{CONCLUSIONES}

Entre las conclusiones, destacamos que:

- El interés y la participación de la comunidad en el proyecto desarrollado fue del $95 \%$; con ello, se logró la identificación de las problemáticas más percibidas por la comunidad en materia ambiental. Como aspecto importante se resalta que las respuestas obtenidas en su gran porcentaje corresponden a personas que han vivido en la zona por más de cinco años, por lo cual pueden identificar con mayor precisión los aspectos que los afectan.

- Los aspectos ambientales que la comunidad identifica que tienen mayor incidencia en la zona son la alta sensación térmica y la falta de suficiente arborización. Adicional a los dos aspectos anteriores, se reconoce el problema de olores ofensivos, inadecuada disposición de residuos sólidos y existe gran preocupación en la zona por el estado de caños, canales y alcantarillas.

- La comunidad reconoce la importancia de incluir en la zona, proyectos de educación ambiental y un gran porcentaje de los encuestados aceptaría participar en talleres de sensibilización ambiental.

AGRADECIMIENTOS: Se agradece la participación en el proyecto a: la Fundación Universitaria Tecnológico Comfenalco, profesor Henry Lambis, el joven investigador Cristian Herrera, los semilleristas de Ciclo Iy II, Ingenio Ambiental de los Programas Ambientales. 


\section{REFERENCIAS}

Amérigo, M., Aragonés, J., De Frutos, B., Sevillano, V. \& Cortés, B. (2007). Underlying dimensions of ecocentric and anthropocentric environmental beliefs. The Spanish Journal of Psychology, 10(1), 97103.

Colmenares A., (2012). Voces y Silencios: Revista Latinoamericana de Educación, 3(1), 102-115.

Cummins, R.A. (2000). Objective and subjective quality of life: An interactive model. Social Indicators Research, 52, 55-72.

Dúran-Muriel, D. (2015). Ministerio de Educación Nacional. Obtenido de Boletín Nº 9. Educación Superior en cifras.

http://www.mineducacion.gov.co/1621/articles350451_recurso_9.pdf.

González, A. \& Amérigo, M. (1999). Actitudes hacia el medio ambiente y conducta ecológica. Psicothema, 11(1), 13-25.

Huppert, F., \& Whittington, J., (2003). Evidence for the independence of positive and negative well being: Implications for quality of life. British Journal of Health psychology, 8, 107-122.

Jiménez O., (2008). Índice de confort de la vegetación. Revista nodo, 3(5), 49-70.

Martínez A., (2005). Ruido por tráfico urbano: conceptos, medidas descriptivas y valoración económica. Revista economía \& Administración, 2(1), 1-49.

Misteli, M.; Angarica, L.; y Ortiz, R. (2009). Manual de Monitoreo y Evaluación Participativos. La Habana, Cuba: Programa de Innovación Agropecuaria Local (PIAL).
Moreno-Jiménez, A.; Fernández-García, F. (2003). El confort climático en los entornos residenciales de las capas altas, medias y bajas de la Comunidad de Madrid: otra forma de desigualdad socioespacial. La distribución espacial de la renta en la Comunidad de Madrid. Análisis y aplicaciones. Madrid: Instituto de Estadística, Consejería de Economía e Innovación Tecnológica. Disponible también en:

http://hdl.handle.net/10486/668622

Sintov, N. D. \& Prescott, C. A. (2011). The influence of social desirability and item priming effects on reports of proenvironmental behavior. Ecopsychology, 3(4), 257-267.

Tréllez E., (2006). Algunos elementos del Proceso de Construcción de la educación ambiental en América Latina. Revista Iberoamericana De Educación, (41), 69-81.

Wrosch, C., \& Scheier, M. (2003). Personality and quality of life: the importance of optimism and goal adjustment. Quality of life Research, 12, 59-72. 